\title{
Measurement of the underlying event using track-jets with the CMS experiment
}

\author{
Paolo Gunnellini*广 \\ Deutsches Elektronen Synchrotron (DESY), Notkestrae 85, 22607 Hamburg (Germany) \\ E-mail: paolo.gunnellini@desy.de
}

\begin{abstract}
A measurement of the underlying event (UE) activity in proton-proton collisions is performed using events with charged-particle jets produced in the central pseudorapidity region $\left(\left|\eta^{\text {jet }}\right|<2\right)$ and with transverse momentum $1 \leq p_{\mathrm{T}}^{\text {jet }}<100 \mathrm{GeV}$. The analysis uses a data sample collected with the CMS experiment at the LHC at a centre-of-mass energy of $2.76 \mathrm{TeV}$, corresponding to an integrated luminosity of $0.3 \mathrm{nb}^{-1}$. The UE activity is measured as a function of $p_{\mathrm{T}}^{\text {jet }}$ in terms of the average multiplicity and scalar sum of transverse momenta $\left(p_{\mathrm{T}}\right)$ of charged particles, with $|\eta|<2$ and $p_{\mathrm{T}}>0.5 \mathrm{GeV}$, in the azimuthal region transverse to the highest $p_{\mathrm{T}}$ jet direction. By further dividing the transverse region into two regions of smaller and larger activity, respectively, various components of the UE activity are separated. The measurements are compared to various model predictions of several Monte Carlo event generators, providing constraints on the theoretical modelling of the UE dynamics.
\end{abstract}

The XXIII International Workshop on Deep Inelastic Scattering and Related Subjects

April 27 - May 1, 2015

Southern Methodist University

Dallas, Texas 75275

*Speaker.

${ }^{\dagger}$ on behalf of the CMS Collaboration. 


\section{Introduction}

Hadron production in high-energy proton-proton $(p p)$ collisions originates from multiple scatterings of the partonic constituents of the protons at central rapidities, and from "spectator" partons emitted in the very forward direction. Such hadron-hadron collisions, in which a "hard" 2-to-2 parton scattering has occurred, are modelled by standard QCD Monte Carlo event generators by means of several components. The "hard scattering" component of the event consists of particles that result from the hadronisation of the two outgoing partons (i.e. the primary outgoing two "jets") plus the particles that arise from initial- and final-state radiation. The Underlying Event (UE) consists of the beam-beam remnants (BBR) and particles that arise from multiple parton interactions (MPI). The BBR are what is left over after a parton is knocked out of each of the initial two beam hadrons. MPI are additional "soft" or "semi-hard" 2-to-2 parton-parton scatterings that occur within the same hadron-hadron collision. An accurate understanding of the UE is required for precise measurements of standard model processes at high energies and searches for new physics. The topological structure of $p p$ interactions with a hard scattering can be used to define experimental observables sensitive to the UE. In $p p$ collisions at the LHC, various experiments have carried out UE measurements at $\sqrt{s}=0.9$ and $7 \mathrm{TeV}$ using events containing a leading (highest $p_{\mathrm{T}}$ ) charged-particle jet [1,2] or a leading charged particle [3, 4], and in DY events [5].

Recent UE measurements [6] extended the usual UE analysis strategy in order to disentangle the different contributions coming from parton shower, MPI and BBR. This is performed by dividing the transverse region in two parts, according to their charged particle content (see Figure 1). One defines the region with the largest (smallest) number of charged particles or scalar transverse momentum sum as "Trans MAX" ("Trans MIN"). For events with large initial- or final-state radiation the region often contains a third jet, while both the "Trans MAX" and "Trans MIN" regions receive contributions from the MPI and BBR components. Thus, while the "Trans MAX" region is affected by the whole UE, the "Trans MIN" region is mainly sensitive only to the MPI and BBR components.

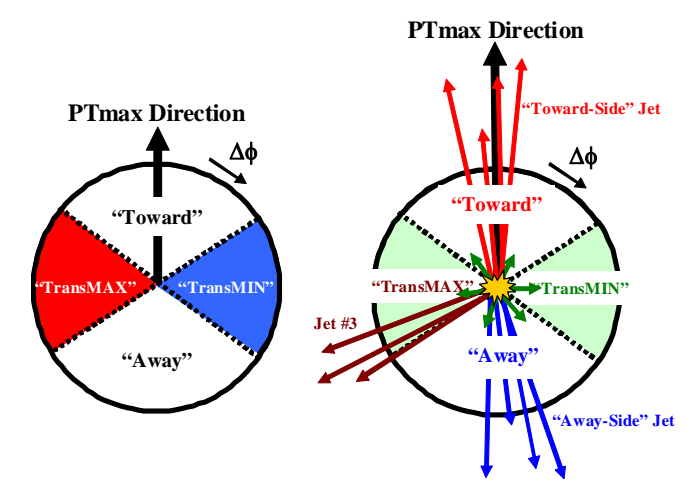

Figure 1: Illustration of correlations in azimuthal angle $\Delta \phi$ relative to the direction of the leading charged particle jet in the event, $p_{\mathrm{T}} \max$.

In the following report, the UE activity is studied by measuring the average multiplicity $\left(N_{c h}\right)$ and scalar transverse momentum sum $\left(\Sigma p_{\mathrm{T}}\right)$ densities of charged particles in the "Trans MIN" and 
"Trans MAX" regions, in $p p$ collisions at $\sqrt{s}=2.76 \mathrm{TeV}$. Comparisons of data to predictions from various Monte Carlo (MC) event generators and tunes are crucial for testing our understanding of the UE components. In this analysis, several event generators and tunes are used for comparison with the data. These are the PYTHIA 6 tune Z2* [7] and tune CUETP6S1 [8], PYTHIA 8 tune 4C [9] and CUETP8S1 [8], and HERWIG ++ 2.7 tune UE-EE-5C [10]. These event generators and tunes differ in the treatment of initial- and final-state radiation, hadronisation, colour reconnections, and cutoff values for the MPI mechanism. These tunes were obtained from comparisons between predictions and data aimed at providing a reasonable description of existing UE data, especially those measured with LHC $p p$ collision. However, minimum bias data from the Tevatron, collected at lower centre-of-mass energies, were also used to obtain the PYTHIA 6 tune CUETP6S1 and the PYTHIA 8 tune CUETP8S1.

\section{Event selection and systematic effects}

The present analysis [11] is performed with a data sample of $p p$ collisions collected with the CMS detector at $\sqrt{s}=2.76 \mathrm{TeV}$ during a dedicated run in March 2011, corresponding to an integrated luminosity of $0.3 \mathrm{nb}^{-1}$. In $6.2 \%$ of the events, there is an extra (pileup) $p p$ collision, corresponding to an average of 0.12 overlapping $p p$ collisions. Minimum bias events were recorded by requiring activity in both beam scintillation counters of the CMS experiment in coincidence with signals from the beam timing detectors in both directions. To reduce the statistical uncertainty for the highly prescaled minimum bias trigger at large $p_{\mathrm{T}}^{\text {jet }}$, single-jet triggers based on information from the calorimeters, with $p_{\mathrm{T}}$ thresholds at 20 and $40 \mathrm{GeV}$, were also used to collect data. Events identified as originating from beam-halo background were removed from the triggered events. The event selection requires exactly one primary vertex with more than four degrees of freedom (approximately 4 particles) and located no more than $\pm 10 \mathrm{~cm}$ from the centre of the luminous region (beamspot) in the z-direction.

For each selected event, the reconstructed track collection needs to be "cleaned up" from undesired tracks, namely secondaries and background from track combinatorics and beam halo associated tracks. Tracks not corresponding to actual charged particles (misreconstructed tracks) are reduced by imposing quality selection criteria [12]. Secondary decays are reduced by requiring that the impact parameter significance $d_{0} / \sigma\left(d_{0}\right)$ (measure of distance between the track and the primary vertex in the $x y$-plane) and the significance in the $z$-direction $d_{z} / \sigma\left(d_{z}\right)$, to be each less than 3. In order to remove tracks with poor momentum measurement, we require the relative uncertainty in the momentum measurement $\sigma\left(p_{\mathrm{T}}\right) / p_{\mathrm{T}}$ to be less than $5 \%$. The average reconstruction efficiency for the selected tracks is about $85 \%$ which drops to $75 \%$ for tracks with $p_{\mathrm{T}}<1 \mathrm{GeV}$ and $|\eta| \approx 2$, while the track misreconstruction rate is about $2 \%$, increasing to about $8 \%$ for tracks with $p_{\mathrm{T}}<1 \mathrm{GeV}$ and $|\eta| \approx 2$.

The event energy scale and reference direction, for the identification of the UE sensitive region, are defined using leading "track jets" [13] also called charged-particle jets. These jets are reconstructed from tracks with $p_{\mathrm{T}}>0.5 \mathrm{GeV}$ and $|\eta|<2.5$ using the SISCone [14] algorithm with distance parameter of 0.5 . From all reconstructed track jets with $|\eta|<2$ and $p_{\mathrm{T}}>1.0 \mathrm{GeV}$, the one with the largest $p_{\mathrm{T}}^{\text {jet }}$ is selected. Only events containing at least one track jet fulfilling these criteria are considered for this analysis. Jets are reconstructed with a matching efficiency of $80 \%$ 
at $p_{\mathrm{T}}^{\text {jet }} \approx 1 \mathrm{GeV}$ and up to $95 \%$ for $p_{\mathrm{T}}^{\text {jet }}>20 \mathrm{GeV}$. Trigger conditions are chosen to keep the trigger efficiency as uniform as possible and close to $100 \%$.

The UE observables $\left(N_{c h}\right.$ and $\left.\Sigma p_{T}\right)$ are reconstructed from selected tracks, with $p_{\mathrm{T}}>0.5 \mathrm{GeV}$ and $|\eta|<2$, in the transverse region to the leading track-jet. These measured observables are corrected for detector effects and selection efficiencies to reflect the primary charged-particle activity. An iterative unfolding technique [15] is used based on 4-dimensional response matrices that correlate the generated and reconstructed level observables. These matrices are constructed from the UE observables as a function of $p_{\mathrm{T}}^{\text {jet }}$ for MC generated particle and reconstructed tracks based on simulated events from the PYTHIA $6 \mathrm{Z} 2$ tune, accounting for the response of the observables to detector effects and inefficiencies.

Several sources of systematic uncertainties affect the results. These include the implementation of the simulation of track and vertex selection criteria, tracker alignment and material content, background contamination, trigger conditions, and pileup contributions. The systematic uncertainties have been estimated and have been added in quadrature to the statistical uncertainties. Their contribution is summarized in Table 1 .

Table 1: Summary of the systematic uncertainties (in percentage) due to various sources. The range is the systematic uncertainty across different $p_{\mathrm{T}}$ values. They are the same for both observables.

\begin{tabular}{|r|c|}
\hline Source & Systematic (\%) \\
\hline \hline Unfolding procedure & $1-4$ \\
\hline Impact parameter signif. & $2-4$ \\
\hline Fraction of misrec. tracks & $0.4-0.5$ \\
\hline Track selection & 0.2 \\
\hline Material density & 1.0 \\
\hline Dead channels & 0.1 \\
\hline Tracker alignment & $0.2-0.3$ \\
\hline Interaction point pos. & 0.2 \\
\hline \hline Total & $2.5-5.8$ \\
\hline
\end{tabular}

\section{Results and comparison to various predictions}

In this Section, the results unfolded to the stable particle level are shown and compared to various MC predictions. In Fig. 2, the charged particle and $\Sigma p_{\mathrm{T}}$ densities after unfolding are shown as a function of $p_{\mathrm{T}}^{\mathrm{jet}}$ in the transverse region in the "Trans MAX" and "Trans MIN" regions.

In the "Trans MIN"region, the amount of UE activity is roughly half of that in the "Trans MAX"region. The $p_{\mathrm{T}}^{\text {jet }}$ dependence observed in the two regions is also quite different. At high- $p_{\mathrm{T}}$, the distributions show a slow rise in the "Trans MAX"region, while for "Trans MIN"the plateaulike region is more pronounced. The data are compared with various MC predictions, obtained with PYTHIA 6, PYTHIA 8 and HERWIG++. In general, all considered MC tunes predict 

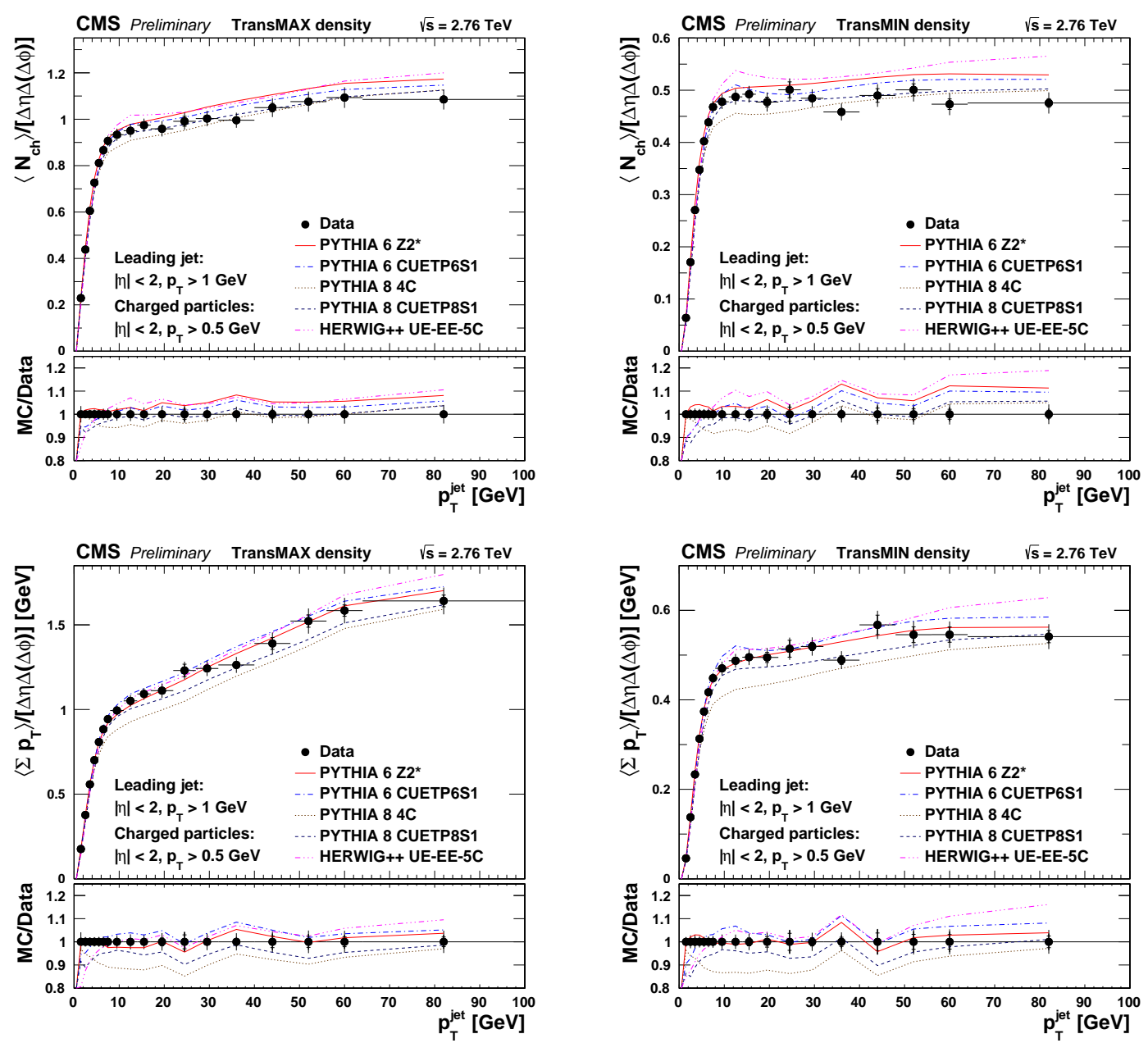

Figure 2: Measured (top) particle density, and (bottom) $\Sigma p_{\mathrm{T}}$ density, in the transverse region relative to the leading charged-particle jet in the event $\left(|\eta|<2,60^{\circ}<|\Delta \varphi|<120^{\circ}\right)$, as a function $p_{\mathrm{T}}^{\text {jet }}$ in the "Trans MAX"(left) and "Trans MIN"(right) regions. The data are compared to various MC simulations. The ratios of MC simulations to the measurements are shown in the bottom panels.

the distinctive change of the amount of activity as a function of the leading jet $p_{\mathrm{T}}$, except for the PYTHIA $84 \mathrm{C}$ which undershoots the data for jet $p_{\mathrm{T}}>5 \mathrm{GeV}$. The latest PYTHIA 6 (PYTHIA 8) tune CUETP6S1 (CUETP8S1) improves the description of the data in comparison with previous tunes: $\mathrm{Z} 2 *$ and $4 \mathrm{C}$. The best description of the data is achieved by PYTHIA 8 CUETP8S1.

Figure 3 shows the charged particle and the $p_{\mathrm{T}}$ sum densities as a function of the $p_{\mathrm{T}}^{\text {jet }}$ in the transverse regions for various collision energies. By comparing data taken at $\sqrt{s}=0.9,2.76$, and $7 \mathrm{TeV}$, a strong growth of the UE activity with the increase of centre-of-mass energy is also observed for the same value of the leading charged-particle jet $p_{\mathrm{T}}$ as expected from the higher parton densities probed at low- $x$ in the protons, and the larger phase space available for parton radiation. All considered tunes are able to correctly predict the UE measurement at the different energies. 

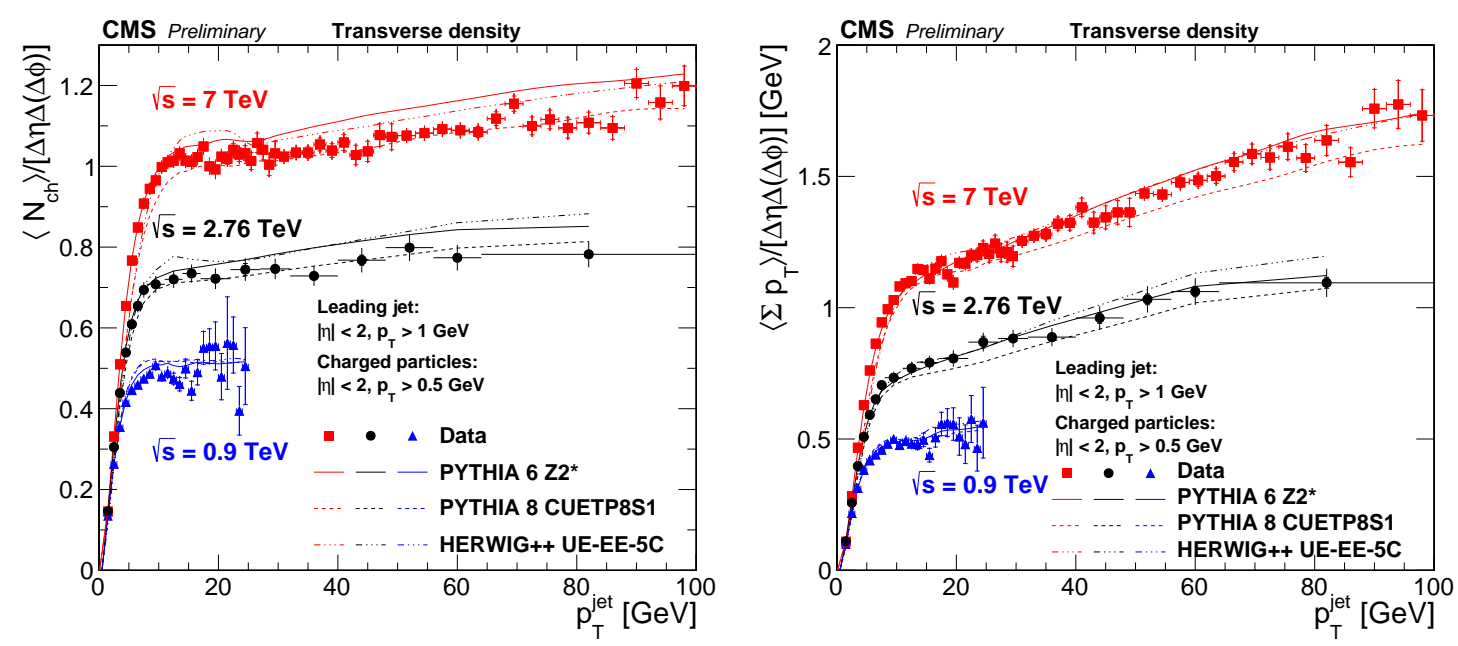

Figure 3: Measured (left) particle density, and (right) $\Sigma p_{\mathrm{T}}$ density, in the transverse region relative to the leading charged-particle jet in the event $\left(|\eta|<2,60^{\circ}<|\Delta \varphi|<120^{\circ}\right)$, as a function $p_{\mathrm{T}}^{\text {jet }}$ at various collision energies: $0.9,2.76$, and $7 \mathrm{TeV}$. The data are compared to various MC simulations.

\section{Conclusions}

The measurement of the underlying event activity in proton-proton collisions at $\sqrt{s}=2.76$ $\mathrm{TeV}$, corresponding to an integrated luminosity of $0.3 \mathrm{nb}^{-1}$, has been presented using events with a charged-particle jet produced at central pseudorapidity $\left(\left|\eta^{\text {jet }}\right|<2\right.$ ) with transverse momenta $1<$ $p_{\mathrm{T}}<100 \mathrm{GeV}$. This analysis complements the results of previous similar measurements at $\sqrt{s}=0.9$ and $7 \mathrm{TeV}$. A steep rise of the underlying activity in the transverse region is seen with increasing leading jet $p_{\mathrm{T}}$. This fast rise is followed by a levelling above jet $p_{\mathrm{T}} \approx 8 \mathrm{GeV}$, with nearly constant particle and small $\Sigma p_{\mathrm{T}}$ density increase. Such a distinct pattern confirms the impact parameter picture of $p p$ collisions featuring an increasing number of MPI for increasing overlap followed by a saturation of hadron production once the hardest most-central collisions are reached. The results are compared to recent tunes of PYTHIA and HERWIG++ Monte Carlo event generators. PYTHIA 6, PYTHIA 8, and HERWIG++ tunes describe the data within 5 to $10 \%$. All MC tunes correctly predict the collision energy dependence of the hadronic activity that is very similar to that observed in the data. This measurement confirms the validity of the latest Monte Carlo generator tunes to describe UE data and adds confidence to the prediction of UE activity at higher collision energies.

\section{References}

[1] S. Chatrchyan et al. [CMS Collaboration], "Measurement of the underlying event activity at the LHC with $\sqrt{s}=7 \mathrm{TeV}$ and comparison with $\sqrt{s}=0.9 \mathrm{TeV}$," JHEP 1109 (2011) 109 [arXiv:1107.0330 [hep-ex]].

[2] G. Aad et al. [ATLAS Collaboration], "Measurement of the underlying event in jet events from $7 \mathrm{TeV}$ proton-proton collisions with the ATLAS detector," Eur. Phys. J. C 74 (2014) 8, 2965

[arXiv:1406.0392 [hep-ex]]. 
[3] V. Khachatryan et al. [CMS Collaboration], "First Measurement of the underlying event activity at the LHC with $\sqrt{s}=0.9$ TeV,” Eur. Phys. J. C 70 (2010) 555 [arXiv:1006.2083 [hep-ex]].

[4] G. Aad et al. [ATLAS Collaboration], "Measurement of underlying event characteristics using charged particles in pp collisions at $\sqrt{s}=900 \mathrm{GeV}$ and $7 \mathrm{TeV}$ with the ATLAS detector," Phys. Rev. D 83 (2011) 112001 [arXiv:1012.0791 [hep-ex]].

[5] S. Chatrchyan et al. [CMS Collaboration], "Measurement of the underlying event in the Drell-Yan process in proton-proton collisions at $\sqrt{s}=7$ TeV,” Eur. Phys. J. C 72 (2012) 2080 [arXiv:1204.1411 [hep-ex]].

[6] CMS Collaboration, "Measurement of the underlying event activity at the LHC at $7 \mathrm{TeV}$ and comparison with 0.9 TeV," CMS-PAS-FSQ-12-020.

[7] S. Chatrchyan et al. [CMS Collaboration], "Study of the underlying event at forward rapidity in pp collisions at $\sqrt{s}=0.9,2.76$, and 7 TeV,” JHEP 1304 (2013) 072 [arXiv:1302.2394 [hep-ex]].

[8] CMS Collaboration, "Underlying event tunes and double parton scattering," CMS-PAS-GEN-14-001.

[9] R. Corke and T. Sjostrand, "Interleaved parton showers and tuning prospects," JHEP 1103 (2011) 032 [arXiv:1011.1759 [hep-ph]].

[10] M. H. Seymour and A. Siodmok, "Constraining MPI models using $\sigma_{\text {eff }}$ and recent Tevatron and LHC underlying event data," JHEP 1310 (2013) 113 [arXiv:1307.5015 [hep-ph]].

[11] CMS Collaboration, "Measurement of the underlying event activity using charged particle jets in proton-proton collisions at 2.76 TeV," CMS-PAS-FSQ-12-025.

[12] S. Chatrchyan et al. [CMS Collaboration], "Description and performance of track and primary-vertex reconstruction with the CMS tracker,” JINST 9 (2014) 10, P10009 [arXiv:1405.6569 [physics.ins-det]].

[13] CMS Collaboration, "Performance of jet reconstruction with charged tracks only," CMS-PAS-JME-08-001.

[14] G. P. Salam and G. Soyez, "A practical seedless infrared-safe cone jet algorithm,” JHEP 0705 (2007) 086 [arXiv:0704.0292 [hep-ph]].

[15] G. D’Agostini, “A multidimensional unfolding method based on Bayes' theorem,” Nucl. Instrum. Meth. A 362 (1995) 487. 